\title{
Association study of dimethylarginine dimethylaminohydrolase 2 gene polymorphisms and coronary heart disease
}

\author{
AI-GUO XU ${ }^{1}$, RONG-MEI XU², CHANG-QING LU ${ }^{3}$, DAN-DAN LI ${ }^{1}$, QIN-FU XU ${ }^{1}$, \\ $\mathrm{JING} \mathrm{GUO}^{1}, \mathrm{XIN} \mathrm{FU}^{1}$, WEI ZHAO ${ }^{1}$ and MENG-YING YAO ${ }^{1}$

\begin{abstract}
${ }^{1}$ Department of Respiratory and Critical Care Medicine, The First Affiliated Hospital of Zhengzhou University, Zhengzhou, Henan 450052; ${ }^{2}$ Human Science Laboratory, Institute of Physical Education of Henan Polytechnic University, Jiaozuo, Henan 454000; ${ }^{3}$ Department of Laboratory Medicine, The Second Affiliated Hospital, Henan University of Traditional Chinese Medicine, Zhengzhou, Henan 450052, P.R. China
\end{abstract}

Received May 30, 2012; Accepted August 14, 2012

DOI: $10.3892 / \mathrm{mmr} .2012 .1038$

\begin{abstract}
While a number of genetic and environmental risk factors for coronary heart disease (CHD) have been identified, the list of potential risk factors remains long. One candidate is dimethylarginine dimethylaminohydrolase (DDAH2), which is known to be polymorphic in humans. The gene product indirectly increases the endogenous production of nitric oxide, an anti-atherogenic molecule. Therefore, alterations in DDAH2 activity may indirectly result in an increased risk of CHD. We studied allele and genotype distributions for two polymorphic loci of $D D A H 2$, rs805305 and rs2272592, in 180 patients with CHD and 180 healthy controls. Disease history and other clinical data were recorded. Polymerase chain reactionrestriction fragment length polymorphism (PCR-RFLP) was used to determine the genotype at rs805305, and ligase detection reaction (LDR) was used to determine the genotype at rs2272592. Systolic blood pressure and blood triglyceride and glucose levels were higher, and history of hypertension, diabetes, smoking and alcohol use was more common in the patients with $\mathrm{CHD}(\mathrm{P}<0.05)$. However, the genotype and allele frequencies at the two polymorphic loci of $D D A H 2$ were not statistically different between the two groups. Therefore, no association was observed between the $D D A H 2$ polymorphisms at rs805305 and rs2272592 and CHD.
\end{abstract}

\section{Introduction}

Coronary heart disease (CHD) occurs with high incidence and results in many deaths from ischemic events (1). CHD may be attributed to the interaction of genetic and environmental

Correspondence to: Dr Ai-Guo Xu, Department of Respiratory and Critical Care Medicine, The First Affiliated Hospital of Zhengzhou University, 1 Jianshedong Road, Zhengzhou, Henan 450052, P.R. China

E-mail: zhengxuag@126.com

Key words: coronary heart disease, dimethylarginine dimethylaminohydrolase, polymorphism factors (2). CHD pathogenesis proceeds when vascular endothelial injury leads to blood lipid deposition in the smooth endarterium, causing the formation of white atherosclerotic plaques. These plaques continue to grow, forming a thrombus that may either block blood flow directly or dislodge and travel through the circulation. These actions may result in myocardial infarction or stroke.

While a number of genetic and environmental factors that promote CHD have been identified, relatively few factors are known to reduce the risk of the disease. One, nitric oxide (NO), is considered to be an endogenous anti-atherosclerotic plaque factor (3). NO is produced in the body by nitric oxide synthase (NOS) (4,5). NOS activity, and therefore NO synthesis, may be reduced by an endogenous competitive inhibitor, asymmetric dimethylarginine (ADMA) (6). ADMA, however, becomes inactivated via hydrolysis by dimethylarginine dimethylaminohydrolases (DDAHs); DDAH has two subtypes, including DDAH2 which is mainly expressed in vascular endothelial cells (7). Changes in DDAH activity or expression may affect the synthesis of NO by affecting ADMA levels (8). DDAH activity and/or expression may be affected by polymorphisms within the gene; therefore, polymorphisms in $D D A H$ may be associated with CHD.

Two polymorphisms in $D D A H$ have been identified: rs805305 and rs2272592 $(9,10)$. In the present study, we determined the frequency of these polymorphisms in CHD patients and healthy individuals to investigate whether these polymorphisms may predispose an individual to CHD.

\section{Materials and methods}

Participants. A total of 180 patients who were diagnosed in The First Affiliated Hospital of Zhengzhou University from October 2008 to May 2011 were enrolled in the study. CHD was confirmed by coronary angiography. The study population included 96 males and 84 females, aged 37-87 years (mean age $54.37 \pm 11.05$ years). Another 180 healthy individuals who received a physical examination in our hospital from January 2010 to June 2011 were selected as a control group; these included 100 males and 80 females, aged $35-87$ years (mean age $52.36 \pm 14.25$ years). No statistically significant difference was 


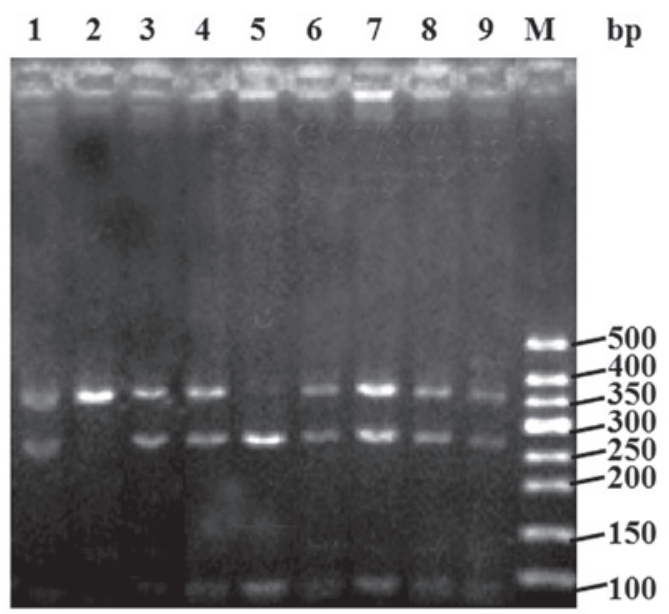

Figure 1. Genotype identification results for the dimethylarginine dimethylaminohydrolase (DDAH2) gene at the rs805305 locus. Lanes 1, 3, 4, 6, 7, 8 and 9, CG; lane 2, GG; lane 5, CC.

observed in age or gender between the two groups. History of hypertension, diabetes, smoking and alcohol use was surveyed for patients in the two groups; the body mass index (BMI) and blood glucose, triglyceride and cholesterol levels were also measured for the two groups. The study was approved by the ethics committee of the First Affiliated Hospital of Zhengzhou University. All patients provided informed consent.

DDAH2 genotype identification at the rs805305 locus. The polymerase chain reaction-restriction fragment length polymorphism (PCR-RFLP) method was used to genotype DDAH2 at the rs805305 locus. A 5-ml sample of venous blood was taken from each patient and genomic DNA was isolated from each sample by phenol-chloroform-isoamyl alcohol extraction. The target fragment that potentially contained the polymorphism was amplified by PCR using the following primers (Takara, Dalian, China): forward, 5'-CCTTCTCGTTCGGGTATTCAG-3'; and reverse, 5'-TCCAGACCTTCCGCTCCT-3'. The reaction mix included $2 \mu \mathrm{l}$ genomic DNA, $2.5 \mu \mathrm{l}$ 10X PCR buffer (Promega, Madison, WI, USA), $1.5 \mu 125 \mathrm{mM}$ dNTP (Promega), $0.25 \mu \mathrm{l}$ 2.5 U/ml Taq DNA polymerase (Promega), $1.5 \mu 125 \mathrm{mM} \mathrm{MgCl}_{2}$ (Promega), $0.5 \mu \mathrm{l}$ forward primer (100 pmol/l), $0.5 \mu \mathrm{l}$ reverse primer (100 pmol/l) and water (to a final volume of $25 \mu \mathrm{l})$. The thermal cycling conditions were as follows: pre-denaturation at $94^{\circ} \mathrm{C}$ for $5 \mathrm{~min} ; 30$ cycles of denaturation at $94^{\circ} \mathrm{C}$ for $60 \mathrm{sec}$, annealing at $58^{\circ} \mathrm{C}$ for $60 \mathrm{sec}$ and extension at $72^{\circ} \mathrm{C}$ for $60 \mathrm{sec}$; and elongation at $72^{\circ} \mathrm{C}$ for $5 \mathrm{~min}$. The amplified PCR product ( $2 \mu \mathrm{l}$ ) was digested with $2 \mu \mathrm{l} \mathrm{SmaI}$ (Promega) in $2 \mu \mathrm{l} 10 \mathrm{X}$ buffer (with deionized water to a final volume of $20 \mu \mathrm{l}$ ) in a $30^{\circ} \mathrm{C}$ water bath for $4 \mathrm{~h}$. The reaction product $(5 \mu \mathrm{l})$ was separated by agarose gel electrophoresis. The expected band sizes are as follows (see Fig. 1): the GG genotype (no SmaI restriction site) produces one band of $341 \mathrm{bp}$; the $\mathrm{CC}$ genotype product produces two bands, 254 and $87 \mathrm{bp}$; and the CG genotype produces 3 bands, 341, 254 and $87 \mathrm{bp}$.

DDAH2 genotype identification at the rs2272592 locus. The ligase detection reaction (LDR) (11) was used to detect the

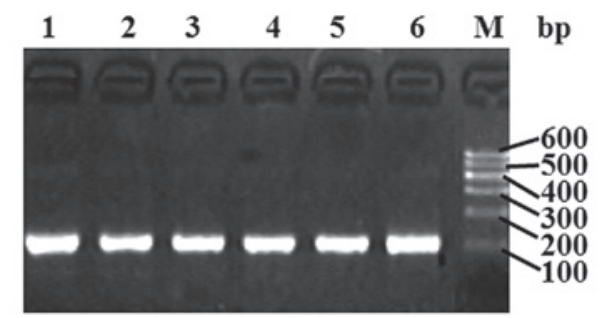

Figure 2. Electrophoretogram of the PCR products of the dimethylarginine dimethylaminohydrolase (DDAH2) gene at the rs2272592 locus. Electrophoresis results of the PCR products of the DDAH2 gene at the rs2272592 locus, with length of $98 \mathrm{bp}$.

target gene sequence containing the SNP. The sequence of the forward primer was 5'-GGGTGGGATGAGTAGGACAA-3', and that of the reverse primer was 5'-ACTGACCCACCC CTCATTC-3'. The reaction mix components were the same as for the preceding reaction. The thermal cycling conditions were as follows: $94^{\circ} \mathrm{C}$ for $5 \mathrm{~min} ; 30$ cycles of $94^{\circ} \mathrm{C}$ for $45 \mathrm{sec}$, $56^{\circ} \mathrm{C}$ for $45 \mathrm{sec}$ and $72^{\circ} \mathrm{C}$ for $45 \mathrm{sec}$; and $72^{\circ} \mathrm{C}$ for $5 \mathrm{~min}$. The products were verified by agarose gel electrophoresis (see Fig. 2). The products were then subjected to LDR with the following probe sequences: rs2272592 reverse_modify, P-TTTAAAGTGTGGGCGACATGGAATGTTTTTTTTTTT TTTTTTTTTTTTT-FAM; rs2272592 reverse_C, TTTTTT TTTTTTTTTTTTTTTTTTATCAAGTGTTTTTACGCAT GGGGG, LDR length 98 bp; and rs2272592 reverse_T, TTTTTTTTTTTTTTTTTTTTTTTTTTATCAAGTGTTTT TACGCAATGGGGA, LDR length $100 \mathrm{bp}$. The reaction mix contained $1 \mu \mathrm{l} 1 \mathrm{X}$ buffer, $1 \mu 112.5 \mathrm{pmol} / \mu 1$ each probe mix, $1 \mu 12$ units ligase, $1 \mu \mathrm{l}$ PCR product and water to a final volume of $10 \mu \mathrm{l}$. The thermal cycling conditions were as follows: $95^{\circ} \mathrm{C}$ for $2 \mathrm{~min}$; 35 cycles of $94^{\circ} \mathrm{C}$ for $30 \mathrm{sec}$; and $50^{\circ} \mathrm{C}$ for $2 \mathrm{~min}$. The reaction products were sequenced (ABI sequencer, Foster City, CA, USA), and Genemapper software was used to analyze the genotype at rs2272592. The results revealed three genotypes at this locus, GG, GA and AA.

Statistical analysis. SPSS 13.0 for Windows statistical software was used to perform statistical analysis. A $\chi^{2}$ test was applied to numerical data and a t-test was applied to measurement data. $\mathrm{P}<0.05$ was considered to indicate a statistically significant result.

\section{Results}

Comparison of medical history and biochemical indexes between the CHD patients and healthy individuals. Analysis of the medical histories and biochemical indices between the patients with CHD and the healthy individuals revealed higher systolic blood pressure and blood triglyceride and glucose levels in the CHD patients than in the control group; these differences were statistically significant $(\mathrm{P}<0.05)$. Additionally, the percentages of patients with CHD who had a history of hypertension, diabetes, smoking or alcohol use were $56.11,27.22,47.78$ and $52.78 \%$, respectively. Each of these percentages was significantly higher than the corresponding value in the control group $(\mathrm{P}<0.05$; Table I). 
Table I. Clinical history of patients with CHD and healthy individuals.

\begin{tabular}{lccc}
\hline & $\begin{array}{c}\text { Experimental } \\
\text { group }(\mathrm{n}=180)\end{array}$ & $\begin{array}{c}\text { Control } \\
\text { group }(\mathrm{n}=180)\end{array}$ & P-value \\
\hline BMI (kg/m $\left.{ }^{2}\right)$ & $25.36 \pm 3.65$ & $24.11 \pm 3.54$ & $<0.05$ \\
SBP $(\mathrm{mmHg})$ & $131.66 \pm 21.09$ & $127.66 \pm 18.85$ & $>0.05$ \\
DBP (mmHg) & $82.84 \pm 20.87$ & $80.66 \pm 18.45$ & $>0.05$ \\
Triglyceride (mmol/l) & $1.37 \pm 0.12$ & $1.20 \pm 0.13$ & $<0.05$ \\
Cholesterol (mmol/l) & $4.20 \pm 0.42$ & $4.14 \pm 0.38$ & $>0.05$ \\
LDL-C (mmol/l) & $2.46 \pm 0.22$ & $2.45 \pm 0.25$ & $>0.05$ \\
HDL-C (mmol/l) & $1.08 \pm 0.15$ & $1.08 \pm 0.14$ & $>0.05$ \\
Glucose (mmol/l) & $6.02 \pm 1.02$ & $5.61 \pm 0.79$ & $<0.05$ \\
History & & & \\
Hypertension (no/yes) & $79 / 101$ & $108 / 72$ & $<0.05$ \\
Diabetes (no/yes) & $131 / 49$ & $169 / 11$ & $<0.05$ \\
Smoking (no/yes) & $94 / 86$ & $114 / 66$ & $<0.05$ \\
Drinking (no/yes) & $85 / 95$ & $162 / 18$ & $<0.05$ \\
\hline
\end{tabular}

CHD, coronary heart disease; BMI, body mass index; SBP, systolic blood pressure; DBP, diastolic blood pressure; LDL-C, low-density lipoprotein cholesterol; HDL-C, high-density lipoprotein cholesterol.

DDAH2 genotype frequency does not differ at the rs 805305 locus. The allele frequencies for the polymorphisms in $D D A H 2$ at rs805305 were in Hardy-Weinberg equilibrium for the experimental and control groups $(\mathrm{P}>0.05)$. The genotype frequencies of GG, CG and CC were, respectively, 17.78, 65.56 and $16.67 \%$ in patients with CHD and 23.33, 55.56 and $21.11 \%$ in the control group; these distributions were not significantly different. The frequencies of $\mathrm{G}$ and $\mathrm{C}$ alleles were 50.56 and $49.44 \%$ in the experimental group and 51.11 and $48.89 \%$, respectively, in the control group; again, these distributions were not significantly different (Table II).

DDAH2 genotype does not differ at the rs2272592 locus. The allele frequencies for the polymorphisms in DDAH2 at rs2272592 were in Hardy-Weinberg equilibrium for the experimental and control groups $(\mathrm{P}>0.05)$. The genotype frequencies of GG, GA and AA were 63.89, 33.89 and $2.22 \%$, respectively, in the experimental group and 71.11, 27.22 and $1.67 \%$, respectively, in the control group; these differences were not statistically significant. The frequencies of $\mathrm{G}$ and $\mathrm{A}$ alleles were 81.94 and $18.06 \%$, respectively, in the experimental group and 84.72 and $15.28 \%$, respectively, in the control group; again, these distributions were not statistically different (Table III).

\section{Discussion}

Studies have shown that NO, a fat-soluble molecule, is able to rapidly diffuse into target cells, including vascular smooth muscle cells and neurons; additionally, NO is considered to be a messenger molecule with wider effects in the body (12). $\mathrm{NO}$ is produced by endothelial cells and it diffuses into and relaxes vascular smooth muscle cells, causing hemangiectasis
Table II. Dimethylarginine dimethylaminohydrolase $(D D A H 2)$ rs805305 genotype and allele frequency distribution.

\begin{tabular}{lccccccc}
\hline & \multicolumn{3}{c}{ Genotype } & & \multicolumn{2}{c}{ Allele } \\
\cline { 2 - 4 } \cline { 7 - 8 } Group & GG & CG & CC & GG+CG & & G & C \\
\hline Experimental & 32 & 118 & 30 & 150 & & 182 & 178 \\
Control & 42 & 100 & 38 & 142 & & 184 & 176 \\
$\chi^{2}$ & & 3.78 & & 1.16 & & 0.02 \\
P & & $>0.05$ & & $>0.05$ & & $>0.05$ \\
\hline
\end{tabular}

Table III. Dimethylarginine dimethylaminohydrolase (DDAH2) rs2272592 genotype and allele frequency distribution.

\begin{tabular}{lccccccc}
\hline & \multicolumn{4}{c}{ Genotype } & & \multicolumn{2}{c}{ Allele } \\
\cline { 2 - 4 } Group & GG & GA & AA & GA+AA & & G & A \\
\hline Experimental & 115 & 61 & 4 & 65 & & 295 & 65 \\
Control & 128 & 49 & 3 & 52 & & 305 & 55 \\
$\chi^{2}$ & & 2.15 & & 2.09 & & 1.01 \\
P & & $>0.05$ & & $>0.05$ & & $>0.05$ \\
\hline
\end{tabular}

and blood pressure decline (13). Furthermore, NO is able to inhibit smooth muscle cell proliferation by preventing platelet aggregation and reducing the formation and growth of atherosclerotic plaques. Endogenous NO is mainly generated via L-arginine conversion and a reaction catalyzed by NOS (14); defects in the metabolism of NO are considered to contribute to the pathogenesis of CHD.

Due to the ability of the endogenous molecule ADMA to inhibit NOS activity, it is very important in the pathogenesis of CHD (15). However, since ADMA is hydrolyzed by DDAH to L-citrulline and dimethylamine in the body, it may lose its activity. DDAH, therefore, may effectively reduce the concentration of ADMA and increase the activity of NOS, thereby increasing NO synthesis. DDAH has two subtypes, DDAH1, which is mainly expressed in the nervous system, and DDAH2, which is mainly expressed in the cardiovascular system (16). Gene knockouts have shown that a lack of DDAH expression in DDAH null mice results in an increased plasma ADMA concentration and reduced NO concentration, along with significantly increased blood pressure (17). Therefore, individuals with reduced DDAH2 activity may carry a CHD risk factor.

The results of this study demonstrated, as expected, that BMI and blood triglyceride and glucose levels were all significantly higher in patients with CHD than in healthy individuals; similarly, histories of hypertension, diabetes, smoking or alcohol use were all more common among CHD patients. Indeed, hypertension, diabetes, smoking and alcohol use are known risk factors for CHD (18).

However, although DDAH2 has generated interest as a potential risk factor for CHD, our analysis of two polymorphic loci in the $D D A H 2$ gene did not uncover any differences in the distribution of alleles between healthy individuals and 
patients with CHD. Therefore, polymorphism at rs805305 or rs2272592 is not associated with CHD. Notably, a study by Jones et al (19) suggested that polymorphisms in the DDAH2 promoter affected $D D A H 2$ expression in vascular endothelial cells and that polymorphism at the $D D A H 2$ promoter 871 locus was correlated with changes in ADMA levels in the body. However, Maas et al (10) reported no correlation between polymorphisms at the rs805304 or rs805305 loci of the DDAH2 promoter and CHD, supporting our findings. The differences between our findings and those reported by Jones et al may result from two characteristics of our study: a smaller sample size and the possibility that participants in our study may not accurately represent the population distribution of the region.

In conclusion, the results from this study reveal that hypertension, diabetes, smoking and alcohol consumption are all risk factors for CHD, but polymorphisms in DDAH2 at rs805305 and rs2272592 are not associated with CHD.

\section{Acknowledgements}

This study was supported by the Key Science and Technology Project of Henan Province (No. 112102310180).

\section{References}

1. Gaziano TA, Bitton A, Anand S, Abrahams-Gessel S and Murphy A: Growing epidemic of coronary heart disease in lowand middle-income countries. Curr Probl Cardiol 35: 72-115, 2010.

2. Tearney GJ, Regar E, Akasaka T, et al: Consensus standards for acquisition, measurement, and reporting of intravascular optical coherence tomography studies: a report from the International Working Group for Intravascular Optical Coherence Tomography Standardization and Validation. J Am Coll Cardiol 59: 1058-1072, 2012.

3. Kolovou G and Giannakopoulou V: Endothelial nitric oxide synthase gene variants and coronary heart disease. Angiology 63 : $84-85,2012$.

4. Chen X, Niroomand F, Liu Z, Zankl A, Katus HA, Jahn L and Tiefenbacher CP: Expression of nitric oxide related enzymes in coronary heart disease. Basic Res Cardiol 101: 346-353, 2006.

5. Jia CQ, Ning Y, Liu TT and Liu ZL: Association between G894T mutation in endothelial nitric oxide synthase gene and premature coronary heart disease. Zhonghua Liu Xing Bing Xue Za Zhi 26 51-53, 2005 (In Chinese).
6. Celik M, Iyisoy A, Celik T, Yilmaz MI, Yuksel UC and Yaman H: The relationship between L-arginine/ADMA ratio and coronary collateral development in patients with low glomerular filtration rate. Cardiol J 19: 29-35, 2012.

7. Palm F, Onozato ML, Luo Z and Wilcox CS: Dimethylarginine dimethylaminohydrolase (DDAH): expression, regulation, and function in the cardiovascular and renal systems. Am J Physiol Heart Circ Physiol 293: H3227-H3245, 2007.

8. Gad MZ, Hassanein SI, Abdel-Maksoud SM, et al: Assessment of serum levels of asymmetric dimethylarginine, symmetric dimethylarginine and L-arginine in coronary artery disease. Biomarkers, 15: 746-752, 2010.

9. Abhary S, Burdon KP, Kuot A, Javadiyan S, Whiting MJ Kasmeridis N, Petrovsky N and Craig JE: Sequence variation in DDAH1 and DDAH2 genes is strongly and additively associated with serum ADMA concentrations in individuals with type 2 diabetes. PLoS One 5: e9462, 2010.

10. Maas R, Erdmann J, Lüneburg N, et al: Polymorphisms in the promoter region of the dimethylarginine dimethylaminohydrolase 2 gene are associated with prevalence of hypertension. Pharmacol Res 60: 488-493, 2009.

11. Wang YZ, Xiao JH, Liu LG, Ye CY, Shen HY, Xu TM and Zhu KZ: Simultaneous detection of hepatitis B virus genotypes and mutations associated with resistance to lamivudine, adefovir, and telbivudine by the polymerase chain reactionligase detection reaction. Braz J Infect Dis 15: 560-566, 2011.

12. Alasbahi RH and Melzig MF: Forskolin and derivatives as tools for studying the role of cAMP. Pharmazie 67: 5-13, 2012.

13. Houston M: The role of magnesium in hypertension and cardiovascular disease. J Clin Hypertens (Greenwich) 13: 843-847, 2011.

14. Anthony S, Leiper J and Vallance P: Endogenous production of nitric oxide synthase inhibitors. Vasc Med (Suppl 1) 10: S3-S9, 2005.

15. Maas R: Pharmacotherapies and their influence on asymmetric dimethylargine (ADMA). Vasc Med (Suppl 1) 10: S49-S57, 2005.

16. Tatematsu S, Wakino S, Kanda T, et al: Role of nitric oxide-producing and -degrading pathways in coronary endothelial dysfunction in chronic kidney disease. J Am Soc Nephrol 18: 741-749, 2007.

17. Leiper J, Nandi M, Torondel B, et al: Disruption of methylarginine metabolism impairs vascular homeostasis. Nat Med 13: 198-203, 2007.

18. Zand Parsa AF, Ziai $\mathrm{H}$ and Haghighi L: The impact of cardiovascular risk factors on the site and extent of coronary artery disease. Cardiovasc J Afr 23: 197-199, 2012.

19. Jones LC, Tran CT, Leiper JM, Hingorani AD and Vallance P: Common genetic variation in a basal promoter element alters DDAH2 expression in endothelial cells. Biochem Biophys Res Commun 310: 836-843, 2003 